This is the peer reviewed version of the following article: Martínez, RM, Barba, C, Robredo, S, Herraiz, M. Improving enantiomeric resolutions by avoiding peak distortion effects in on - line coupled liquid chromatography to gas chromatography. Chirality. 2019; 31: 879- 891. https:/l. doi.org/10.1002/chir.23121, which has been published in final form at https://doi.org/10.1002/chir.23121. This article may be used foknaifaditithercial purposes in accordance with Wiley Terms and Conditions for Use of Self-Archived Versions.

\title{
Improving enantiomeric resolutions by avoiding peak distortion effects in on-line coupled liquid chromatography to gas chromatography
}

\author{
Rosa M. Martínez, ${ }^{[a]}$ Carmen Barba, ${ }^{[a, b]}$ Sergio Robredo, ${ }^{[c]}$ Marta Herraiz ${ }^{*}[a]$
}

Abstract: In this work we study the effect of different variables affecting elution profile distortion on the enantiomeric resolution eventually achievable when working with on-line coupled liquid chromatography to gas chromatography (LC-GC). Specifically, the proposed configuration combines achiral reversed-phase liquid chromatography (RPLC) and chiral gas chromatography (enantio-GC), with heptakis-(2,3,6-tri-O-methyl)- $\beta$ cyclodextrin as enantioselective stationary phase, to analyse target fractions transferred (from LC to GC) via the through oven transfer adsorption desorption (TOTAD) interface. The high degree of orthogonality resulting from the combination of two chromatographic columns having very different separation mechanisms (and also requiring mobile phases in distinct physical states), as well as integration of the sample preparation step in the first dimension of the system, significantly contributed to exploit the performance of the proposed two-dimensional approach. Occasional adverse effects, which may result in severe peak distortions during LC-GC analysis and could be explained by flow instabilities due to viscous fingering, are circumvented by using the outstanding capacity of the TOTAD interface for achieving effective elimination of the eluent arriving from the LC preseparation.

Keywords: chiral analysis, enantio-multidimensional chromatography, enantiodifferentiation, on-line coupling LC-GC, peak shape distortion, viscous fingering

\section{I INTRODUCTION}

The complexity of a high number of mixtures (as those found, for example, in food, environmental, biological and pharmaceutical sciences) makes it difficult to perform the reliable enantiodifferentiation of specific chiral target compounds whose enantiomeric resolutions may be impossible due to coelutions with other analytes. In such circumstances, not only highly efficient separation techniques are required to obtain enantiomeric resolutions but also appropriate preseparation methods have often to be carried out prior to the chromatographic analysis itself.

In contrast to conventional (one-dimensional, 1D) chromatography, two-dimensional (2D) chromatographic techniques offer a clearly superior performance in terms of peak capacity $\left(n_{c}\right)$, that is, the maximum number of peaks that can be separated with resolution unity, in a given time interval, during a single chromatographic analysis. ${ }^{1,2}$ On-line 2D chromatographic techniques can be divided into two groups, namely, heart cutting and comprehensive, depending on the amount of the column effluent that is directed from the first to the second dimension. When working with heart cutting (as it is the case in GC-GC and LC-LC) only those selected parts of the effluent containing the target compounds are transferred to the second dimension ${ }^{3-7}$ while comprehensive techniques (e.g., GCxGC and LCxLC) eventually provide the separation of the complete sample by the two dimensions. ${ }^{8-14}$

Among the analytical approaches included in the first group, the so-called enantioselective multidimensional heartcut GC-GC system (abbreviated as enantio-MDGC) has already proved its usefulness for achieving enantiomeric resolutions of specific compounds in complex mixtures. ${ }^{7}$ The relative simplicity of combining the two involved dimensions evidently arises from the mobile-phase total compatibility, but limitations due to the requirement of having substantially clean samples, containing compounds of high and moderate volatility, must be always kept in mind.

In those cases in which effective sample clean-up steps are demanded prior to the gas chromatographic analysis (to avoid interferences as well as to increase the sensitivity achievable in the overall procedure), the on-line coupling of liquid chromatography and gas chromatography (LC-GC) can be the technique of choice. ${ }^{15-17}$ In this instance, enantioseparation can be accomplished by introducing a chiral GC step, as an additional dimension to achiral LC, in such a way that the fraction containing the target analytes is pre-separated in the achiral column and, thereupon, on-line transferred to the chiral column. Obviously, the combination of the two mentioned techniques within one system can yield a high effective peak capacity as the two dimensions are chromatographically

[a] Instituto de Ciencia y Tecnología de Alimentos y Nutrición (ICTAN) Consejo Superior de Investigaciones Científicas (CSIC) c/ Juan de la Cierva 3, 28006 Madrid, Spain

[b] Present address: Departamento de Tecnología de Alimentos Universidad Pública de Navarra Campus Arrosadía s/n, 31006 Pamplona, Spain

[c] Laboratorio Arbitral Agroalimentario (LAA) Ministerio de Agricultura, Alimentación y Medio Ambiente c/ Aguarón 13, Ctra. de A Coruña, Km 10,700. 28023 Madrid, Spain

\section{Correspondence}

M. Herraiz

Instituto de Ciencia y Tecnología de Alimentos y Nutrición (ICTAN)

Consejo Superior de Investigaciones Científicas (CSIC)

c/ Juan de la Cierva 3, 28006 Madrid, Spain

E-mail: mherraiz@ictan.csic.es 
orthogonal (i.e., the separation mechanisms in each dimension are independent of each other and provide different selectivities). It follows that when combining LC and GC, the orthogonality may be significantly increased with respect to that obtained when coupling either LC to LC or GC to GC. . $^{14,18,19}$

Thus, interfacing LC to GC offers clear advantages concerning the possibility of combining the selectivity of LC with the efficiency and sensitivity of GC, but the fact that the two separation techniques operate in two different physical states involves an unquestionable difficulty. The use of a programmed temperature vaporizer (PTV) as interface of an on-line coupled LC-GC system had previously demonstrated to be useful even for the analysis of aqueous samples ${ }^{20-23}$ although the necessity of promoting solvent elimination, by disconnecting (from the injector body and prior to the LC-GC transfer) the column housed into the GC, initially hindered the automation of the overall procedure. This drawback was overcome through the development of the TOTAD (through oven transfer adsorption desorption) interface, ${ }^{24-25}$ based on a modified PTV injector, which exhibits an outstanding ability to eliminate polar eluents, so that automated analysis (even using reversed-phase (RP) mode in the LC step) can be performed with high sensitivity and selectivity. ${ }^{26-30}$

Occasionally, however, the occurrence of strongly distorted peaks has been observed during experimental work with online coupled LC-GC but the origin of such problem has not been so far investigated.

Considering both the inherent high orthogonality of a multidimensional system combining LC with GC and the fact that the effectiveness of enhanced peak capacities in achieving better resolutions also depends on the separation conditions and the properties of the analytes, ${ }^{14,18,19}$ the objective of this work was to assess the potential risk of elution profile distortion when using an on-line coupled LC-GC system in which the columns in the two dimensions are connected via the TOTAD interface. We also intended to study the way of preventing peak deterioration to finally obtain the desirable chromatographic resolution (and, when required, the enantiodifferentiation) of target compounds.

To this aim, the possible occurrence of viscous fingering (due to a viscosity mismatch) and the subsequent severe peak shape distortion when interfacing LC to GC were also investigated.

\section{I MATERIALS AND METHODS}

\subsection{Samples and chemicals}

Methanol and water (both of HPLC grade) were used as mobile phase in the LC step of the on-line LC-GC analysis. Both eluents were purchased from Lab Scan LTD (Gliwice, Poland). Absolute ethanol (WWR International S.A.S., Fontenay-sous-Bois) was used as solvent for the test mixtures. Racemic $\gamma$-lactones, namely, rac- $\gamma$-hexalactone $\left(\mathrm{C}_{6}\right)$, rac- $\gamma$-octalactone $\left(\mathrm{C}_{8}\right)$, rac- $\gamma$ nonalactone $\left(\mathrm{C}_{9}\right)$, rac- $\gamma$-decalactone $\left(\mathrm{C}_{10}\right)$, rac- $\gamma$-undecalactone $\left(\mathrm{C}_{11}\right)$ and rac- $\gamma$-dodecalactone $\left(\mathrm{C}_{12}\right)$ were provided by Aldrich (St. Louis, MO, USA).

\section{2 | On-line coupled LC-GC}

The analyses were conducted using an on-line LC-GC equipment, fitted with a TOTAD interface, which allows full automatic operation. The HPLC system (Konik model 560) consisted of a quaternary pump, a manual sample introduction valve (model 7725 , Rheodyne, California, USA) having a $250-\mu \mathrm{L}$ volume loop and an ultraviolet (UV) detector operated at $205 \mathrm{~nm}$. The gas chromatograph (Konik, model HRGC 4000B) was equipped with a FID detector. Data acquisition and processing were performed with KoniKrom Plus (Konik, Sant Cugat del Vallés, Barcelona, Spain).

\subsection{LC conditions}

LC preseparations were accomplished on a $100 \mathrm{~mm} \times 4.6 \mathrm{~mm}$ i.d. $\mathrm{C}_{4}$, Hypersil, $10 \mu \mathrm{m}$ column (Phenomenex, Torrance, California, USA), which was kept at $45^{\circ} \mathrm{C}$. All analyses were carried out using methanol/water $(90: 10 ; \mathrm{v}: \mathrm{v})$ as the mobile phase at a flow rate of $1.8 \mathrm{~mL} \mathrm{~min}{ }^{-1}$. Upon elution of the beginning of the target fraction, the flow rate was reduced to 0.1 $\mathrm{mL} \mathrm{min}^{-1}$ and after completion of the transfer step it was raised again up to $1.8 \mathrm{~mL} \mathrm{~min}{ }^{-1}$. The elution time of the LC fraction to be transferred to the gas chromatograph was fixed by injecting into the LC equipment a $250 \mu \mathrm{L}$-volume of a solution (in ethanol) containing $100 \mathrm{mg} \mathrm{L}^{-1}$ of each standard (i.e., $\gamma$-hexalactone, $\gamma$ octalactone, $\gamma$-nonalactone, $\gamma$-decalactone, $\gamma$-undecalactone and $\gamma$-dodecalactone). Afterwards, other solutions of the same test mixture (containing 10,1 or $0.1 \mathrm{mg} \mathrm{L}^{-1}$ of each standard in ethanol) were injected into LC and the corresponding target fractions were then transferred to GC.

\subsection{Interface operation mode}

A porous polymer, based on 2,6-diphenyl-p-phenylenoxid (Tenax TA, 80-100 mesh) provided by Supelco (Bellefonte, PA, USA), was used to pack the glass-liner $(110 \mathrm{~mm} \times 2 \mathrm{~mm}$ i.d. $\times 3 \mathrm{~mm}$ o.d.) placed inside the body of the TOTAD interface. A $1-\mathrm{cm}$ length of this packing material, which was plugged at both ends with glass wool was introduced into the liner and then conditioned under a helium stream by successively increasing the temperature $10^{\circ} \mathrm{C}$ every $5 \mathrm{~min}$ from $40^{\circ} \mathrm{C}$ to $350^{\circ} \mathrm{C}$. The final temperature was held for $60 \mathrm{~min}$.

The interface operation mode includes five stages, specifically, a) stabilisation, b) transfer, c) solvent elimination, d) thermal desorption and e) cleaning. Initially, the TOTAD interface was stabilised at $75^{\circ} \mathrm{C}$ and maintained at this temperature until completion of the solvent elimination step. The test mixtures were injected using the aforementioned LC manual injection valve. To reach the liner, the injected samples were propelled by the LC pump operated at the established flow rate. The six-port valve, placed immediately after the UV detector of the LC equipment, was automatically switched in the precise moment in which the front of the target fraction began to elute. Subsequently, this fraction was directed to the interface of the LC-GC system as depicted in Figure 1 with a simplified scheme of the TOTAD interface during the LC to GC transfer step. As shown, a helium flow rate, noted as (8), was used to drive the effluent coming from LC through the packing material (Tenax TA) while the solvent was vented to waste through a stainless steel tubing. In this way, the analytes are retained on the packing material placed in the liner while the solvent is eliminated. Upon completion of the transfer step, the eluent arriving from the LC preseparation was sent to waste by switching again the earlier mentioned six-port valve (named as (3) in Figure 1). Afterwards, the TOTAD interface was quickly heated (up to the final temperature established for each analysis) to achieve the thermal desorption of the retained analytes, which were at that time pushed by the helium stream and transferred to the GC 
column to start the GC analysis. Between consecutive runs the interface was cleaned by heating up to $350^{\circ} \mathrm{C}$ for $10 \mathrm{~min}$ (while keeping the helium flow) and then it was cooled to $75^{\circ} \mathrm{C}$ so that another analysis could be started.

Further details on the TOTAD interface original design as well as on the recently modified sealing system design are given elsewhere. ${ }^{24,25,29}$ Also, more aspects concerning the TOTAD interface operation mode are discussed below in the context of the results obtained successively throughout the experimentation performed in this study.

\subsection{GC analysis}

Gas chromatographic separations of the analytes eluted from LC and transferred to GC through the TOTAD interface were carried out on a Chirasil- $\beta$-Dex (Varian, Middelburg, The Netherlands) fused-silica capillary column $(25 \mathrm{~m} \times 0.25 \mathrm{~mm}$ i.d. $)$ coated with a $0.25 \mu \mathrm{m}$ film thickness of permethylated $\beta$-CD (i.e., heptakis$(2,3,6$-tri-O-methyl)- $\beta$-cyclodextrin) with helium as the carrier gas (column head pressure: $110 \mathrm{kPa}$ ). In all analyses, the initial column temperature was maintained throughout the interface operation mode (thus including transfer, solvent elimination and thermal desorption) and, ultimately, the GC analysis was started by increasing the oven temperature as detailed below for each particular case. The separation was monitored via FID.

\section{I RESULTS AND DISCUSSION}

\subsection{General considerations}

Although previous experimentation with on-line coupled LC-GC has sporadically shown strange and inexplicable chromatograms with seriously distorted peaks, nobody knows for certain what the origin of the problem is and, hence, nobody has established so far the experimental conditions under which such strange peak profiles could be avoided. Moreover, even the manifestation of this type of distorted peaks has, as yet, received little attention from chromatographers working with multidimensional techniques and, particularly, it has been overlooked (or neglected) in the literature concerning on-line coupling LC-GC via the TOTAD interface.

For these reasons, the starting point of our study was to investigate possible causes of those peak distortion effects having the potential to damage and even ruin an analysis as they eventually result in unacceptable chromatographic resolutions.

A further point to be considered was the fact that when working with a two-dimensional chromatographic system (as the on-line coupled LC-GC), the attainable orthogonality between the two dimensions not only depends on their separation mechanisms, but also on separation conditions and sample characteristics. Hence, analysis in both dimensions must be carried out by carefully choosing those interdependent parameters affecting the experimentation and, additionally, taking into account the physicochemical properties of sample constituents. Obviously, it must also be kept in mind that operating conditions in the interface used to connect both LCand GC-columns should not contribute to the deterioration of the separation finally achievable.

This study was performed with standard mixtures of relatively low volatile compounds (at different concentrations) in order to assess the possibility of optimizing the orthogonality of the technique for further potential applications to the enantiodifferentiation of chiral compouns occurring in difficult samples. Thus, we evaluated the effect of LC, GC and interface analytical conditions on the separation achieved in each experimental run. To this aim, we chose a mixture of racemic $\gamma$ - lactones (with boiling points and molecular weights varying from 219 to $295^{\circ} \mathrm{C}$ and from 114 to 198 , respectively) because of two reasons: a) chiral lactones are present in many complex samples such as food and beverage aroma and b) their enantiomeric resolution may be problematic due to possible peak defocusing which can adversely affect peak capacity and separation efficiency of a multidimensional chromatographic system.

In this respect, it should be emphasized that our intention was not to develop a new method to separate chiral lactones but to examine the peak shape profile resulting from different experimental conditions with a view to improve chromatographic resolutions and, particularly, to eventually achieve a reliable enantiodifferentiation of chiral compounds by on-line coupled LC-GC.

\subsection{First dimension (LC step)}

For operating in the first dimension we initially considered different sets of chromatographic conditions including stationary phase, mobile phase, column efficiency and sample capacity.

Concerning the relative polarities of both stationary and mobile phases used in the LC preseparation, it is clear that typical normal phase conditions (i.e., polar stationary phases and apolar eluents) are somewhat easy to use in a coupled LC-GC system, since apolar eluents are suitable for GC. Moreover, the relatively low volume of vapour generated during evaporation of an apolar eluent makes it easier the transfer from LC to GC.16,17 However, we decided to work with the much more demanding coupling which involves the use of reversed phase conditions (i.e., apolar stationary phases and polar eluents) because a very high percentage of separations performed in LC uses, precisely, reversed phase operation mode (and, interestingly, the compatibility with water-based eluents is of relevant importance when analysing a high number of complex samples such as foods, beverages or biological ones). Precisely for this reason, we aimed to evaluate peak shape distortion types when interfacing RPLC to GC.

Considering the potential application of our study in achieving the enantiodifferentiation of chiral compounds occurring in complex samples (through the integration of sample preparation in the overall analysis) the role that we assigned for the LC-step in our two-dimensional system was to accomplish the selective clean-up, concentration and/or fractionation required when a direct analysis was to be intended. Accordingly, in this particular case the LC step should provide better clean-up than conventional sample preparation procedures to separate the target analytes from the matrix compounds and, consequently, high separation efficiency of the LC-column was not considered to be essential.

Furthermore, the choice of LC columns having high sample capacities was regarded as a good option for the determination of minor enantiomers occurring at trace levels in samples containing large amounts of disturbing components. As the sample capacity increases with rising column internal diameter (and also increases the sensitivity of the whole analysis), a column with an internal diameter of $4.6 \mathrm{~mm}$ was expected to be a good compromise between sufficient column efficiency for the LC preseparation step and desirable sample capacity. The fact that the larger the internal diameter of the LC column, the higher is the flow-rate (and, thereby, the larger is the volume of the peak to be transferred to GC) was expected to be a manageable problem since, as mentioned above, the TOTAD interface had already proved its capability to efficiently eliminate high vapour volumes of polar eluents and, therefore, to handle water-based mobile phases. As, in this particular case, the eluent from LC is transferred to $\mathrm{GC}$ at $0.1 \mu \mathrm{L} / \mathrm{min}, 7.2 \mathrm{~min}$ is the time required to complete this step, namely, the transfer of the target fraction $(0.72 \mathrm{~mL})$ coming from the preseparation performed in LC.

All in all, our study focused on the use of a $4.6 \mathrm{~mm}$ i.d. $\mathrm{C}_{4}$ column and RPLC conditions (methanol/water as eluent of the system) as detailed in the Materials and Methods section. 


\section{3 | Transfer step: TOTAD interface}

As Figure 1 shows a schematic representation of the TOTAD interface operation mode during the transfer step, the valves are positioned to allow the fraction previously selected in the LCpreseparation to be transferred to the interface body. At this stage, the carrier gas (helium) enters the interface through both the oven side, named as (8), and the opposite side, indicated as (9). In the first case, the helium flow drives the effluent coming from LC through both glass wool and the porous polymer (i.e., Tenax TA) placed inside the interface. Moreover, once the target solutes have been retained in the packing material, this helium flow also pushes the remaining solvent to be sent to waste. On the other hand, the carrier gas stream, noted as (9) in Figure 1, circulates outside the packed liner and is used to prevent condensation of the remaining solvent while is being directed to waste.

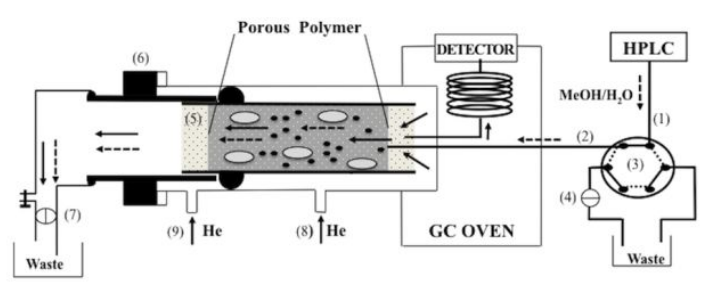

FIGURE 1 Schematic illustration of the TOTAD interface operation mode during the transfer step. Valves are positioned for driving the target fraction (arriving from the LC-preseparation through the sixport valve) to the interface body. (1) Stainless steel tubing to transfer from LC to six-port valve; (2) Silica capillary tubing; (3) Sixport valve; (4) Electrovalve; (5) Glass wool; (6) Heated cover; (7) Electrovalve; (8) Helium stream entering the packing material inside the liner of the TOTAD interface through the GC oven side; (9) Auxiliary helium flow applied through the opposite GC oven side; (dotted arrows) liquid flow; (solid arrows) gas flow; (grey ellipse) solvent from LC; (small black ellipse) analytes retained on the packing material

Bearing in mind that the evident incompatibility of mobile phases used in LC and GC plays an important role regarding the orthogonality of the multidimensional platform, we considered the efficient removal of the solvent arriving from the LCpreseparation step as a critical point of the overall procedure. In fact, not only the original design of the TOTAD interface but also the previously mentioned modification of the sealing system design had already addressed this aspect to avoid GC-column flooding (and its subsequent irreversible deterioration) so that the possibility of achieving the effective solvent elimination (even when transferring aqueous eluents) has already been claimed in the reported literature.

Then, we first set a helium flow rate to be applied for a specific time (including both transfer step itself and also an additional period) to promote the elimination of the remaining solvent in the liner of the TOTAD interface. However, the unexpected (and inconsistent) results discussed below showed the necessity of studying in depth the way in which some experimental variables may affect the chromatographic resolution (and, in this particular case, the enantiodifferentiation of chiral compounds).

With this objective, two helium flow-rates $\left(200 \mathrm{~mL} \mathrm{~min}^{-1}\right.$ and $1000 \mathrm{~mL} \mathrm{~min}-1$ ) were set for solvent removal during different times (4 and $15 \mathrm{~min}$ ) to evaluate their effects on the system orthogonality. Equally, various temperatures (250, 290 and 350 $\left.{ }^{\circ} \mathrm{C}\right)$ were applied for different times $(5,15,20$ and $30 \mathrm{~min})$ to accomplish the thermal desorption of the compounds retained in the interface.

\subsection{Second dimension (GC step)}

Firstly we established the experimental conditions under which the analysis should be run by considering those parameters that influence the peak capacity, namely, compatibility of the involved dimensions and orthogonality. As the GC part of our twodimensional system provides the final analytical step which, in this case, must lead to the enantiodifferentiation of the target chiral compounds, we used a permethylated $\beta$-cyclodextrin (detailed in subsection 2.5) as enantioselective stationary phase, which is recommended for the enantiomeric separation of a wide range of chiral compounds. ${ }^{31}$ Nevertheless, as detailed below, various types of severe peak shape deterioration were observed throughout the experimentation in several chromatograms recorded in the second dimension and, consequently, the orthogonal separation effect obtained in these cases was not acceptable.

\subsection{On-line coupled enantio-(LC-GC) analysis}

Figure 2a shows the fraction selected to be transferred from the liquid chromatograph to the gas chromatograph in each RPLC$\mathrm{GC}$ analysis. As can be seen, the fraction containing the target analytes (in this work, racemic lactones) eluted between 0.64 and $1.04 \mathrm{~min}$, which means that a fraction as large as $0.72 \mathrm{~mL}$ had to be transferred from LC to GC (i.e., the fraction corresponding to the volume eluted in $0.40 \mathrm{~min}, 1.8 \mathrm{~mL} \mathrm{~min}{ }^{-1}$ being the flow-rate applied in the LC preseparation). Throughout the experimental work, always the same fraction was selected and thereupon transferred from the first to the second dimension to perform each individual enantio-(LC-GC) analysis.

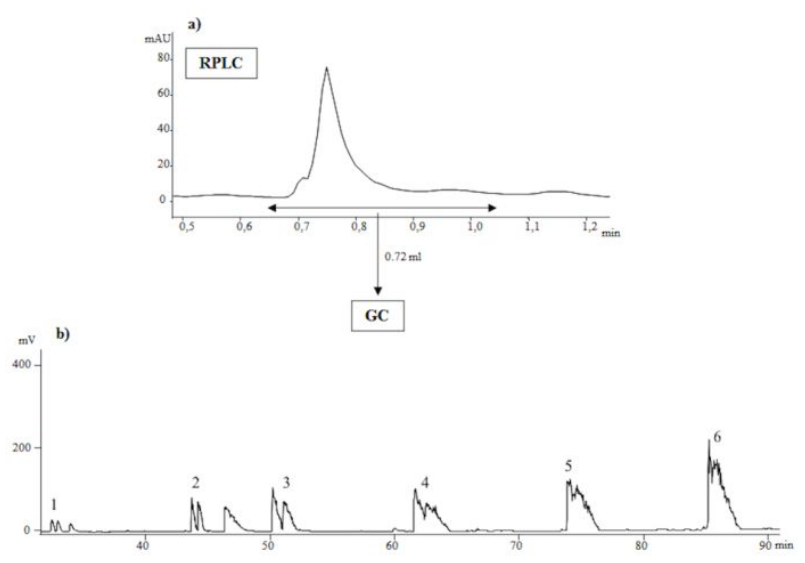

FIGURE 2 On-line coupled RPLC-GC analysis: (a) LC chromatogram resulting from the injection of a $250-\mu \mathrm{L}$ volume of the test mixture. The fraction to be transferred from $L C$ to $G C$ is indicated by a double arrow. Methanol/water 90:10 (v:v) was used as eluent (flow-rate: $1.8 \mathrm{~mL} \mathrm{~min}^{-1}$ ); (b) GC separation of the LC to $\mathrm{GC}$ transferred fraction selected from the test mixture $\left(10 \mathrm{mg} \mathrm{L}^{-1}\right.$ of each chiral lactone in ethanol). Column temperature $80^{\circ} \mathrm{C}$ for 18.7 $\mathrm{min}$, to $130^{\circ} \mathrm{C}\left(2^{\circ} \mathrm{C} \mathrm{min}-1\right)$ for $15 \mathrm{~min}$ and to $180^{\circ} \mathrm{C}\left(1^{\circ} \mathrm{C} \mathrm{min}-1\right)$ for $60 \mathrm{~min}$. Identification peak number: (1) $(R)$ - and $(S)-\gamma$-hexalactone, (2) $(R)$ - and $(S)-\gamma$-octalactone, (3) $(R)$ - and $(S)-\gamma$-nonalactone, (4) $(R)$ - and $(S)-\gamma$-decalactone, (5) $(R)$ - and $(S)-\gamma$-undecalactone, (6) $(R)$ - and $(S)-\gamma$-dodecalactone. (See text for further details) 
The GC separation shown in Figure $2 b$ was obtained using a helium flow-rate of $200 \mathrm{~mL} \mathrm{~min}^{-1}$ (for $4 \mathrm{~min}$ ) for solvent removal and starting the column temperature program at $80^{\circ} \mathrm{C}$ (which was ramped up to $180^{\circ} \mathrm{C}$ as detailed in the figure caption).
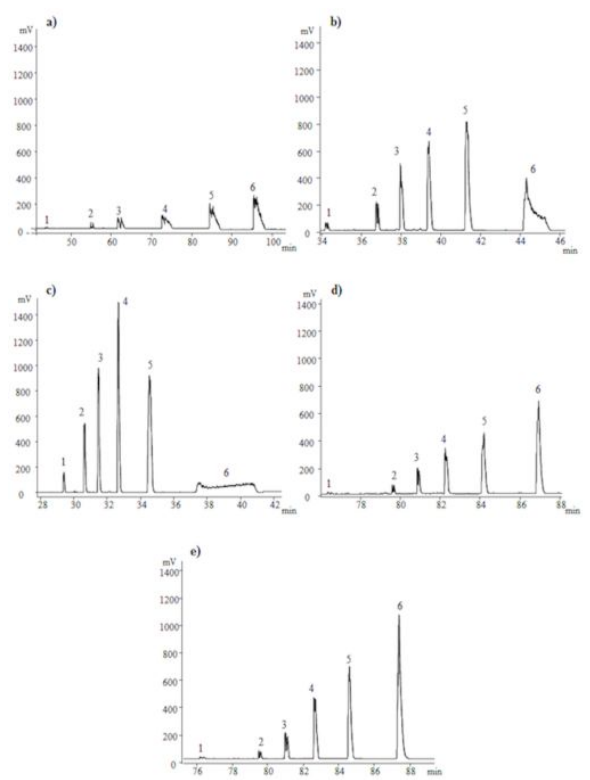

FIGURE 3 Separations obtained in GC when performing the LC $\mathrm{GC}$ transfer of the selected fraction from the test mixture $\left(10 \mathrm{mg} \mathrm{L}^{-1}\right.$ of each chiral lactone in ethanol) under different experimental conditions: Helium flow-rate applied for solvent removal during transfer step: $1000 \mathrm{~mL} \mathrm{~min}^{-1}$ (15 min) in Figures 3a, 3d and 3e; 200 $\mathrm{mL} \min ^{-1}(4 \mathrm{~min})$ in Figures $3 \mathrm{~b}$ and $3 \mathrm{c}$. Column temperature $(\mathbf{a}): 80$ ${ }^{\circ} \mathrm{C}$ for $18.7 \mathrm{~min}$, to $130^{\circ} \mathrm{C}\left(2^{\circ} \mathrm{C} \mathrm{min}-1\right)$ for $15 \mathrm{~min}$ and to $180^{\circ} \mathrm{C}(1$ ${ }^{\circ} \mathrm{C} \mathrm{min}^{-1}$ ) for $60 \mathrm{~min}$. Column temperature (b): $40^{\circ} \mathrm{C}$ for $23.7 \mathrm{~min}$

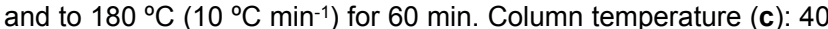
${ }^{\circ} \mathrm{C}$ for $23.7 \mathrm{~min}$ and to $180^{\circ} \mathrm{C}\left(25^{\circ} \mathrm{C} \mathrm{min}^{-1}\right)$ for $60 \mathrm{~min}$. Column temperature (d): $40^{\circ} \mathrm{C}$ for $49.7 \mathrm{~min}$, to $80^{\circ} \mathrm{C}\left(2{ }^{\circ} \mathrm{C} \mathrm{min}-1\right)$ for $1 \mathrm{~min}$ and to $180^{\circ} \mathrm{C}\left(10^{\circ} \mathrm{C} \mathrm{min}-1\right)$ for $60 \mathrm{~min}$. Column temperature (e): 40 ${ }^{\circ} \mathrm{C}$ for $49.7 \mathrm{~min}$, to $80^{\circ} \mathrm{C}\left(2{ }^{\circ} \mathrm{C} \mathrm{min}^{-1}\right)$ for $1 \mathrm{~min}$, to $165^{\circ} \mathrm{C}\left(10^{\circ} \mathrm{C}\right.$ $\left.\mathrm{min}^{-1}\right)$ for $1 \mathrm{~min}$ and to $180^{\circ} \mathrm{C}\left(6^{\circ} \mathrm{C} \mathrm{min}-1\right)$ for $60 \mathrm{~min}$. Identification peak number as in Figure 2

Chromatogram given in Figure $3 a$ was recorded by applying helium flow at $1000 \mathrm{~mL} \mathrm{~min}{ }^{-1}$ (for $15 \mathrm{~min}$ ) for solvent elimination, while the column temperature program (starting at $80^{\circ} \mathrm{C}$ and ramped up to $180^{\circ} \mathrm{C}$ ) was identical to that of Figure $2 \mathrm{~b}$. Since peak distortion and asymmetry observed in both chromatograms $2 \mathrm{~b}$ and $3 \mathrm{a}$ yielded unacceptable results, we first intended to promote a focusing mechanism of the solutes at the head of the second dimension column to avoid or, at least, to minimize peak broadening. Specifically, in Figures $3 b$ and $3 c$ a lower initial temperature (i.e., $40^{\circ} \mathrm{C}$ ) was set to induce cold trapping by temporarily increasing the retention power of the column inlet during sample transfer from Tenax TA (placed inside the TOTAD interface) into the GC-column. In this way, we tried to retard the migration velocity of the compounds entering the GC-column in order to allow those portions of the sample introduced at the end of the transfer period to reach the zone created at the column entrance.

Concerning results shown in Figures 2 and 3, it is interesting to underline that thermal desorption of the compounds retained into the TOTAD interface was intended working at $250{ }^{\circ} \mathrm{C}$ (kept for $5 \mathrm{~min}$ ) in Figures $2 \mathrm{~b}, 3 \mathrm{~b}$ and $3 \mathrm{c}$; at $290^{\circ} \mathrm{C}$ (for $30 \mathrm{~min}$ ) in Figure $3 \mathrm{a}$ and at $290^{\circ} \mathrm{C}$ (for $15 \mathrm{~min}$ ) in Figures $3 \mathrm{~d}$ and $3 \mathrm{e}$. Additionally, it was clear that the initial column temperature should not be rapidly increased (as it was in Figures $3 b$ and $3 c$ ) in order to avoid component release acceleration eventually causing humpbacked peak profiles.

In this context, it should also be emphasized that experimental variables affecting the transfer operation mode in the TOTAD interface must be optimized for each particular analysis. It follows that, if required, a volume vapour high enough to recondensate at the GC-column entrance can also be purposely transferred from LC to GC. Migration of the sample can be then delayed (ideally until completing the transfer step) owed to the retention power of the temporary liquid film formed by the recondensed layer at the GC-column inlet, which finally may result in band focusing (solvent trapping effect).

Particularly, experimental conditions applied in Figures $3 b$ and $3 \mathrm{c}$, which involve column temperature programs from $40^{\circ} \mathrm{C}$ and increased with two different gradients up to $180^{\circ} \mathrm{C}$, resulted in sharpening several peaks, but also in dramatically broadening and distorting the last eluted components. Apparently, $\gamma$ dodecalactone was not chromatographed normally because it did not remain at the column entrance until the sample transfer to the GC column was completed, likely owing to inefficient trapping in the layer of the recondensed solvent. Consequently, $\gamma$-dodecalactone could not be reconcentrated by solvent trapping because it did not migrate noticeably during solvent evaporation so that, in some way, it can be assumed that it was only partially trapped in the recondensed solvent layer.

As previously described by other authors, 2,32 partial solvent trapping in one-dimensional GC analysis may result in characteristic chair and stool shaped peaks. Particularly in the present work, it must be taken into account that peak broadening effect observed in partially trapped components is expected to become more drastic with the very large volumes transferred from LC into GC, as well as with the use of highly polar eluents because they have higher evaporation energies (and, therefore, require longer evaporation times) than apolar eluents.

All in all, it was evident that experimental conditions applied for obtaining Figures $3 \mathrm{~d}$ and $3 \mathrm{e}$ resulted in better symmetric shaped peaks for the eluted compounds.

\subsection{Viscous Fingering}

On the other hand, all throughout the experimentation we occasionally recorded a further type of strongly distorted peaks, which appeared as if there were a mixture of analytes eluting under the same peak, although they had been obtained from a pure standard. Actually, we had been observing such a circumstance for several years, when analyzing very different types of samples, not having been able, as yet, to identify its origin.

When tackling this issue in the present work, experimentation was hindered by various limitations mainly concerning the apparently irreproducible patterns observed in successive analyses for the eluted peak profiles. Trying to explore the cause of this peculiar type of distortion, we defined a wide range of experimental conditions under which a high number of RPLC-GC analyses were performed. Unfortunately, data obtained were confusing and incomprehensible so that they did not lead to a conclusion but gave rise to the supposition that the sources of fluctuations were uncontrolled and occurred randomly.

Conclusively, relevant information was provided by two consecutive analyses which were performed under almost identical experimental conditions. Figure 4 shows peak shape profiles observed for the enantiodifferentiation of both $(R, S)-\gamma$ undecalactone and $(R, S)$ - $\gamma$-dodecalactone, the helium flow rate applied during the transfer step being the most significant difference. When applying a helium flow of $200 \mathrm{~mL} \mathrm{~min}{ }^{-1}$ (maintained for $4 \mathrm{~min}$ ) to promote solvent removal during the transfer to the GC column of the fraction coming from the LCpreseparation, a strangely distorted peak (whose shape profile could not be reproduced) was obtained for the last eluted compound (Figure 4a). However, simply by increasing (from 200 $\mathrm{mL} \mathrm{min}^{-1}$ to $500 \mathrm{~mL} \mathrm{~min}^{-1}$ ) the helium flow-rate applied during the transfer step (in order to ensure a complete solvent elimination in the TOTAD interface) a remarkable difference became apparent (see Figure 4b) as clearly well shaped peaks were observed for both $\gamma$-undecalactone and $\gamma$-dodecalactone. 


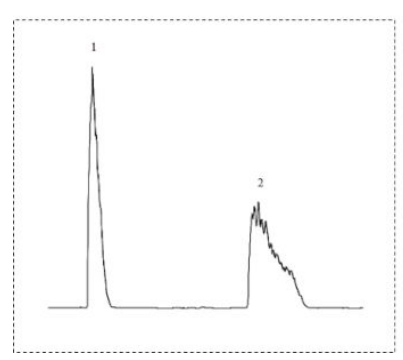

FIGURE 4 Peak shape profiles recorded for the enantiodifferentiation of $(R, S)-\gamma$-undecalactone (noted as 1$)$ and $(R, S)-\gamma$-dodecalactone (2) when applying a helium flow of either $200 \mathrm{~mL} \mathrm{~min}-1$ (4 min) in chromatogram a or $500 \mathrm{~mL} \mathrm{~min}^{-1}(4 \mathrm{~min})$ in chromatogram $\mathbf{b}$, to promote solvent removal during the LC to GC transfer step. See text for further details

Trying to seek an explanation for the mechanism involved in the origin of the type of distortion above mentioned, it is interesting to consider an aspect, namely, viscous fingering, that is usually neglected in multidimensional separations although its potential importance has already been realised by some researchers. ${ }^{14,33}$ Briefly, viscous fingering is a flow instability phenomenon which takes place when two fluids of different viscosities come into contact with each other. This is relevant in chromatography because the mobile phase often does not have the same viscosity as the sample plug (namely, the solute introduced onto the column as a plug of either solvent or vapour). Thus, the destabilisation of the interface between a lowviscosity fluid (e.g., a mobile phase) pushing a more viscous fluid (e.g., a sample plug) can affect the separation performance to an extent that becomes more substantial as the viscosity contrast increases. Concretely, the instability produced when the lowviscosity fluid displaces and penetrates into the high-viscosity fluid may result in a complex, and apparently chaotic, pattern resembling fingers, hence the name of the effect. In such a case, the band shape can be affected so much that elution profiles exhibit signs of peak splitting and peak asymmetry that eventually result in a severely distorted peak behaviour. ${ }^{34-37}$

In fact, the flow instability process of viscous fingering has been studied for several years in different fields but its impact on some chromatographic systems has not been so far thoroughly investigated. As in preparative scale chromatography very large volumes of highly viscous samples are commonly used, a viscosity mismatch between sample plug and mobile phase is easily to be found. That is why viscous fingering in preparative scale separations has already been considered as a phenomenon of interest, but its potential concern when working at analytical scale is usually neglected.

Indeed, conditions required to expect viscous fingering are rarely encountered in analytical separations performed in one dimensional approach because small volumes of diluted samples are typically used so that viscosities of both sample and mobile phase are similar.

However, as in the present study we used a coupled chromatographic separation system requiring the transport of different fluids that may come in contact (and whose mix is inadequate), experimental conditions enhancing flow instability were to be expected and, consequently, eventual manifestations of viscous fingering phenomena should not be excluded. Actually, the occurrence of their adverse effects is to be precisely found when coupling LC to GC inasmuch as the mobile phases used in both dimensions have markedly different viscosities.

Furthermore, the fact of having transferred a $720-\mu \mathrm{L}$ fraction from LC into GC (as detailed in section 3.5) means that, in each experimental run, a very large sample diluted in one mobile phase must be introduced into a second one (what, apart from the obvious difficulty, is advantageous to increase analytical sensitivity). Then, as the volume of solvent transported in the solute plug to the second dimension clearly exceeds sample volumes typically injected into a GC column, the mismatch between mobile phase and sample plug is much more likely to negatively influence the chromatographic performance in comparison to the rather small viscosity differences observed when sampling normal analytical size injection plugs in onedimensional analysis.

This is clarified in Figure 5 with a schematic representation of the TOTAD interface operation mode showing the valves positioned to allow the thermal desorption of the analytes retained on the packing material (Tenax TA) and its subsequent transfer to the column housed into the $\mathrm{GC}$ oven. In comparison to Figure 1, it is clear that helium flow through the GC oven side, noted as (8) in both Figures 1 and 5, was interrupted so that at this stage helium enters into the liner solely through the opposite side, indicated as (9). Consequently, this helium stream (1 $\mathrm{mL} / \mathrm{min}^{-1}$ ) is used to drive the target solutes to the $\mathrm{GC}$ column.

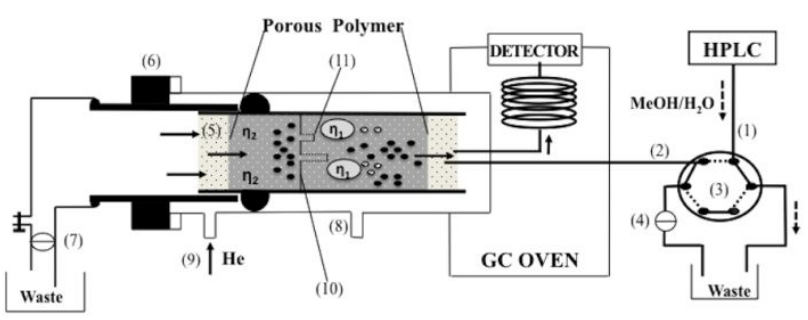

FIGURE 5 Schematic illustration of the TOTAD interface operation mode during the thermal desorption step. Valves are positioned to allow the desorption of the analytes retained on the packing material placed into the TOTAD interface and its subsequent transfer to the column housed into the GC oven. See Figure 1 for symbols (1-9). (10) Interface, in an unperturbed zone, between fluids of viscosities $\eta_{1}$ (in this case $\mathrm{MeOH} / \mathrm{H}_{2} \mathrm{O}$ ) and $\eta_{2}$ (helium); (11) Interface, in a perturbed zone, between fluids of viscosities $\eta_{1}$ and $\eta_{2}$; (grey ellipse) solvent remaining into the packed liner of the TOTAD interface after incomplete solvent elimination; (small black ellipse) compounds being transferred to $\mathrm{GC}$

In our context, the assumption that viscous fingering arises between the interface of two fluids of different viscosity can be explained by considering that the gas (helium; viscosity 0,02 $\mathrm{mPa} \cdot \mathrm{s}$ at $20^{\circ} \mathrm{C}$ ) going through the porous material placed into the liner of the TOTAD interface can finger into the liquid arriving from the LC step (90:10 (v:v) of methanol:water; viscosity 0.84 $\mathrm{mPa} \cdot \mathrm{s}$ at $20^{\circ} \mathrm{C}$ ) as well as into the solvent remaining in the interface after its incomplete elimination (resulting, for example, from inadequate experimental conditions during the transfer step).

In this case an initial perturbation at the interface of these two fluids of viscosities $\eta_{1}$ (methanol:water) and $\eta_{2}$ (helium) can move faster than the main flow providing that the flow velocity in the perturbed section is higher than that of the unperturbed section. This initial perturbation will displace the interface ahead of the original position, thus resulting different interfaces between the two mentioned fluids depending on the way in which the corresponding zones are being affected (see in Figure 5 the representation of the interface between fluids of viscosities $\eta_{1}$ and $\eta_{2}$ in both an unperturbed and a perturbed zone, indicated as (10) and (11), respectively). 
In consequence, if $\eta_{2}<\eta_{1}$, the fluid 2 can protrude through the interface between fluids 1 and 2 and this perturbation can become amplified eventually forming a pattern that resembles a set of fingers. Hence, the fact that during LC to GC transfer the displacing fluid $(\mathrm{He})$ is less viscous than the displaced fluid (methanol:water) can explain the observed flow instability.

It should be pointed out that the physical mechanism of viscous fingering when a fluid is displaced by a less viscous one in a porous medium (concretely, in packed LC columns) as well as possible sources of factors triggering instability and the described type of perturbation (e.g., temperature and pressure changes, among others), have been previously discussed by other authors. ${ }^{34,37,38}$ Nevertheless, the occurrence of viscous fingering (and the way to overcome its detrimental effect on peak separation) has not been so far described in on-line coupled LCGC.

All in all, it seems clear that, in our particular case, the mismatch between the plug of liquid transporting the selected fraction (namely, the heart-cut section coming from LC) and the helium flows applied during both the transfer step and the GC analysis could lead to viscosity differences large enough to cause viscous fingering. Moreover, the successive flow-stream changes required (in each experimental run) from one mobile phase to another as well as the large sample volume transferred from the first to the second dimension may have further enhanced viscous fingering development.

It should be taken into account that, as reported by other authors, ${ }^{38}$ without positive visual proof of the manifestation of viscous fingering is extremely difficult to detect its occurrence. In fact, the obvious experimental limitations encountered in the real observation of the finger development in chromatographic systems have so far restricted the validation of the models proposed to explain the inherent complex pattern formation. In any case, the same authors mentioned that the knowledge and understanding of this phenomenon can be useful to actually enhance separation performance, especially in multidimensional chromatographic techniques. Furthermore, the fact that the development of new multidimensional separation methods tends to create experimental conditions under which viscous fingering will be favored, even at the analytical scale, has also been stressed by other authors. ${ }^{33}$

Thereby, the potential adverse impact of viscous fingering on chromatographic performance should be a key criterion in performing two-dimensional separations because it is evident that, whatever their origin, those effects that can likely decrease the chromatographic resolution of chiral (and achiral) compounds must always be investigated to avoid or minimise performance loss in any two-dimensional separation.

\subsection{Solvent elimination in LC-GC interface}

Figure 6a depicts the GC chromatogram recorded from the test mixture (10 $\mathrm{mg} \mathrm{L}^{-1}$ of each lactone in ethanol) after having transferred the target fraction from LC into GC and, subsequently, promoted the thermal desorption at $350^{\circ} \mathrm{C}$ (for 20 $\mathrm{min}$ ). The enlarged area (Figure 6b) clearly shows that $\gamma$ hexalactone was almost completely lost under these experimental conditions while significant losses were also observed for $\gamma$-octalactone and $\gamma$-nonalactone.

To ensure complete solvent elimination when performing the on-line LC-GC transfer, the same helium flow used in Figure 6 for solvent removal (i.e, $1000 \mathrm{~mL} / \mathrm{min}$ for $15 \mathrm{~min}$ ) was also applied when lower concentrations (i.e., 1 and $0.1 \mathrm{mg} \mathrm{L}^{-1}$ of each lactone in ethanol) were injected (see Figure 7) while increasing the time (up to $30 \mathrm{~min}$ ) at which the temperature during the desorption step $\left(350^{\circ} \mathrm{C}\right)$ was kept. Besides, the GC-column temperature was slightly modified with respect to Figure 6 in order to improve the recovery of the first eluted lactones.

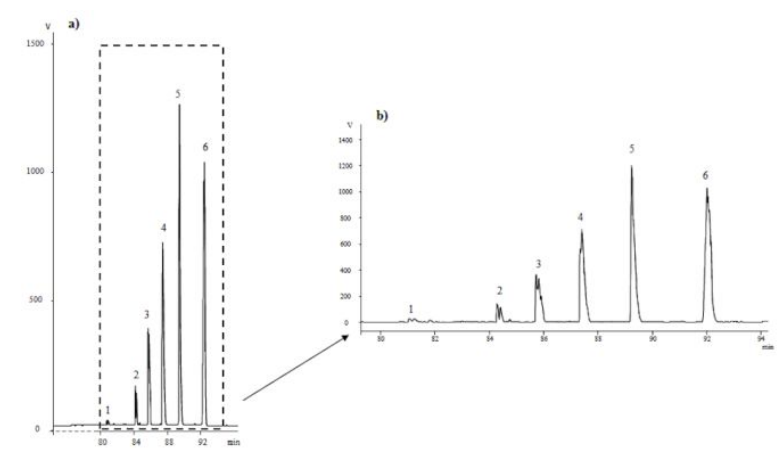

FIGURE 6 Separation obtained in GC when performing the RPLC$\mathrm{GC}$ analysis of the test mixture $\left(10 \mathrm{mg} \mathrm{L}^{-1}\right.$ of each chiral lactone in ethanol). Helium flow-rate applied for solvent removal during transfer step: $1000 \mathrm{~mL} \mathrm{~min}^{-1}(15 \mathrm{~min})$. Column temperature: $40^{\circ} \mathrm{C}$ for $54.7 \mathrm{~min}$, to $80^{\circ} \mathrm{C}\left(2^{\circ} \mathrm{C} \mathrm{min}{ }^{-1}\right)$ for $1 \mathrm{~min}$, to $165^{\circ} \mathrm{C}\left(10^{\circ} \mathrm{C} \mathrm{min}{ }^{-1}\right)$ for $1 \mathrm{~min}$ and to $180^{\circ} \mathrm{C}\left(6^{\circ} \mathrm{C} \mathrm{min}^{-1}\right)$ for $60 \mathrm{~min}$. The dashed-line section (a) has been expanded to show the enantioseparation achieved (b). Identification peak number as in Figure 2

Specifically, the initial temperature was maintained for ten minutes more during the experimental run corresponding to Figure 7 , so that $\gamma$-octalactone and $\gamma$-nonalactone were recovered in higher amounts. Under these experimental conditions, however, the most volatile analyte ( $\gamma$-hexalactone) was again lost (most likely in the interface during removal of the eluent coming from LC).

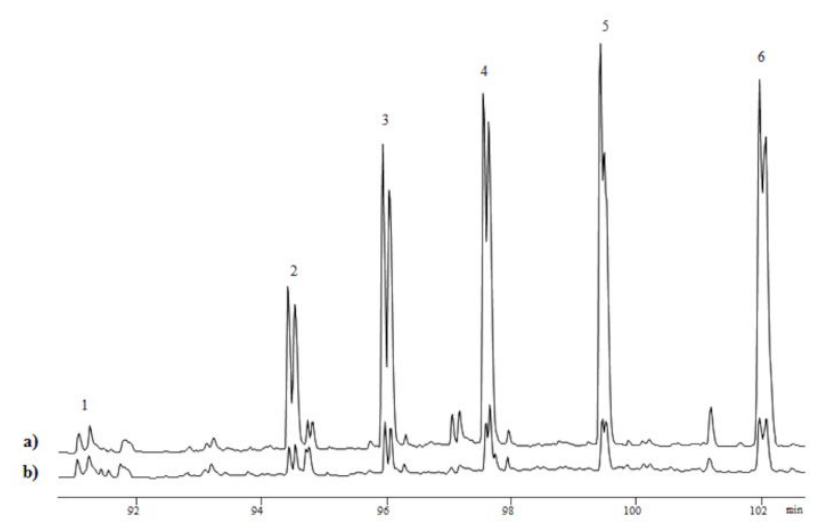

FIGURE 7 Enantiodifferentiation observed in GC when carrying out RPLC-GC analysis of test mixtures containing either $1 \mathrm{mg} \mathrm{L}^{-1}(\mathrm{a})$ or 0.1 $\mathrm{mg} \mathrm{L}^{-1}(\mathrm{~b})$ of each chiral lactone in ethanol. Helium flow-rate applied for solvent removal during transfer step: $1000 \mathrm{~mL} \mathrm{~min}^{-1}$ (15 min). Column temperature: $40^{\circ} \mathrm{C}$ for $64.7 \mathrm{~min}$, to $80^{\circ} \mathrm{C}\left(2{ }^{\circ} \mathrm{C} \mathrm{min}-1\right)$ for 1 $\mathrm{min}$, to $165^{\circ} \mathrm{C}\left(10^{\circ} \mathrm{C} \mathrm{min}^{-1}\right)$ for $1 \mathrm{~min}$ and to $180^{\circ} \mathrm{C}\left(6^{\circ} \mathrm{C} \mathrm{min}-1\right)$ for 60 min. Identification peak number as in Figure 2

Summarizing, it can be stated that the more efficient elimination (in the transfer step) of the very large vapour volumes yielded by the methanol-water eluent used as mobile phase in the first dimension (LC), the less likely viscous fingering 
phenomenon will be observed in the GC step. In fact, the magnitude of the viscous fingering effect depends not only on the above mentioned degree of viscosity mismatch between two fluids but also on relative migration velocities between solute and sample solvent. Certainly, this is also applicable when the TOTAD interface is operated under vacuum conditions as well as when using nitrogen during the solvent elimination step as proposed by other authors. ${ }^{39-41}$ In this way it can be significantly reduced the consumption of the helium needed to remove the eluent resulting from either large volume sampling into GC or direct transfer to $\mathrm{GC}$ of the fraction coming from the preseparation performed in the LC step.

\section{I CONCLUSION}

Although implementation of multidimensional systems may occasionally be difficult, because parameter optimization is a matter of much compromises, their key role when analysing complex samples should always be considered. In comparison to the low or moderate peak capacity values achievable with one-dimensional chromatographic techniques, the increased peak capacities resulting from the use, in a multidimensional platform, of systems that differ significantly in chromatographic selectivities is of great interest. Particularly, when coupling LC to GC via the TOTAD interface, highly selective methods (with high orthogonalities and high efficiencies) are attainable. This is not only due to the fact of involving two dimensions working under mobile phases in distinct physical states and with very different separation mechanisms but also to the possibilities that the TOTAD interface operation mode offers to enhance the overall analytical performance (namely, feasibility of transferring very large volume fractions and compatibility with apolar as well as polar eluents).

Interestingly, the fact that the TOTAD interface allows efficient elimination of even aqueous solvents coming from the preseparation performed in the LC step makes it possible to substantially decrease flow instability phenomena that may result from viscosity differences between mobile phases used in the two dimensions. Accordingly, the consequences of viscous fingering (i.e., severe peak distortion profiles that significantly decreases separation performance) can be prevented to render the two-dimensional system more efficient and viable. Additionally, the capability of integrating the sample preparation step (which is usually required, when using one-dimensional systems, before performing the chromatographic separation) is a further benefit of the on-line LC-GC analysis which must be taken into account when accomplishing the stereodifferentiation of chiral compounds occurring in complex samples.

\section{ACKNOWLEDGEMENTS}

Financial assistance from the Spanish Ministry of Economy and Competitiveness (Projects IPT-010000-2010-017 and DEP2012$35225)$ is gratefully acknowledged.

\section{REFERENCES}

1. Giddings JC. Two-dimensional separations: concept and promise. Anal Chem. 1984;56:1258A-1270A.

2. Poole CF. The Essence of Chromatography. Amsterdam: Elsevier Science BV; 2003. 925 p.

3. Deans DR. Use of heart cutting in gas chromatography: A review. J Chromatogr. 1981;203:19-28.

4. Schomburg G. Two-dimensional gas chromatography: Principles, instrumentation, methods. J Chromatogr A. 1995;703:309-325.

5. Bertsch W. Two-dimensional gas chromatography. Concepts, instrumentation, and applications - Part 1: Fundamentals, conventional two-dimensional gas chromatography, selected applications. HRC-J High Resolut Chromatogr. 1999;22:647-665.

6. Mondello L, Lewis AC, Bartle KD. Multidimensional Chromatography. Chichester, UK: John Wiley \& Sons; 2001. 458 p.

7. Tranchida $P Q$, Sciarrone $D$, Dugo $P$, Mondello L. Heartcutting multidimensional gas chromatography: A review of recent evolution, applications, and future prospects. Anal Chim Acta. 2012;716:66-75.

8. Liu Z, Phillips JB. Comprehensive two-dimensional gas chromatography using an on-column thermal modulator interface. J Chromatogr Sci. 1991;29: 227-231.

9. Phillips JB, Xu JZ. Comprehensive multi-dimensional gas chromatography. J Chromatogr A. 1995;703:327-334.

10. Phillips JB, Beens J. Comprehensive two-dimensional gas chromatography: A hyphenated method with strong coupling between the two dimensions. $J$ Chromatogr $A$. 1999;856:331-347.

11. Bertsch W. Two-dimensional gas chromatography. Concepts, instrumentation, and applications - Part 2: Comprehensive two-dimensional gas chromatography. HRC-J High Resolut Chromatogr. 2000;23:167-181.

12. Marriott PJ, Kinghorn RM. In: Handley AJ, Adlard ER, editors. Gas chromatographic techniques and applications. Sheffield: Academic Press; 2001. 320 p.

13. Marriott P, Shellie R. Principles and applications of comprehensive two-dimensional gas chromatography. TrACTrends Anal Chem. 2002;21:573-583.

14. François I, Sandra K, Sandra P. Comprehensive liquid chromatography: Fundamental aspects and practical considerations - A review. Anal Chim Acta. 2009;641:14-31.

15. Grob K. Efficiency through combining high-performance liquid chromatography and high resolution gas chromatography: Progress 1995-1999. J Chromatogr A. 2000;892:407-420.

16. Hyötyläinen T, Riekkola ML. On-line coupled liquid chromatography - gas chromatography. J Chromatogr A. 2003;1000:357-384.

17. Purcaro G, Moret S, Conte L. Sample pre-fractionation of environmental and foods samples using LC-GC multidimensional techniques. TrAC-Trends Anal Chem. 2013;43:146-160.

18. Giddings JC. Sample dimensionality: A predictor of orderdisorder in component peak distribution in multidimensional separation. J Chromatogr A. 1995;703:163-169.

19. Camenzuli M, Schoenmakers PJ. A new measure of orthogonality for multi-dimensional chromatography. Anal Chim Acta. 2014;838:93-101.

20. Señoráns FJ, Reglero G, Herraiz M. Use of a programmed temperature injector for on-line reversed-phase liquid chromatography-capillary gas chromatography. J Chromatogr Sci. 1995;33:446-450.

21. Blanch GP, Ruiz del Castillo ML, Herraiz M. Enantiomer analysis of chiral lactones in foods by on-line coupled reversedphase liquid chromatography gas chromatography. J Chromatogr Sci. 1998;36:589-594.

22. Ruiz del Castillo ML, Herraiz M, Blanch GP. Determination of the enantiomeric composition of $\gamma$-lactones in edible oils by on-line coupled high performance liquid chromatography and gas chromatography. J Agric Food Chem. 2000;48:1186-1190.

23. Caja MM, Blanch GP, Herraiz M, Ruiz del Castillo ML. Online reversed-phase liquid chromatography-gas chromatography coupled to mass spectrometry for enantiomeric analysis of chiral compounds in fruit beverages. $J$ Chromatogr $A$. 2004; 1054:81-85.

24. Pérez M, Alario J, Vázquez A, Villén J. On-line reversed phase LC-GC by using the new TOTAD (through oven transfer adsorption desorption) interface: Application to parathion residue analysis. J Microcolumn Sep. 1999;11:582-589.

25. Pérez M, Alario J, Vázquez A, Villén J. Pesticide residue analysis by off-line SPE and on-line reversed-phase LC-GC using the through-oven-transfer adsorption/desorption interface. Anal Chem. 2000;72:846-852. 


\section{Chirality}

26. Toledano RM, Cortés JM, Rubio-Moraga A, Villén J, Vázquez A. Analysis of free and esterified sterols in edible oils by online reversed phase liquid chromatography gas chromatography (RPLC-GC) using the through oven transfer adsorption desorption (TOTAD) interface. Food Chem. 2012;135:610-615.

27. Toledano RM, Díaz-Plaza EM, Cortés JM, Blázquez I, Vázquez A, Villén J, Muñoz-Guerra J. Analysis of steroids in human urine by on line liquid chromatography-gas chromatography-mass spectrometry using the through oven transfer adsorption desorption interface and a fraction collector, Anal Chim Acta. 2012;741:78-85.

28. Cortés JM, Andini JC, Toledano RM, Quintero C, Villén J, Vázquez A. Analysis of polychlorinated biphenyls in transformer oils by automated on-line coupling reversed phase liquid chromatography-gas chromatography using the through oven transfer adsorption desorption (TOTAD) interface. Int $J$ Environ Anal Chem. 2013;93:461-471.

29. Martínez RM, Herraiz M, Santa-María G, Barba C. On-line coupled reversed phase liquid chromatography and gas chromatography: a new sealing design for the TOTAD interface. Talanta. 2014;120:23-29.

30. Barba C, Martínez RM, Calvo MM, Santa-María G, Herraiz M. Chiral analysis by online coupling of reversed-phase liquid chromatography to gas chromatography and mass spectrometry. Chirality. 2012;24:420-426.

31. Juza M, Schurig V. The analytical separation of enantiomers by gas chromatography on chiral stationary phases. In: DettmerWilde K, Engewald W, editors. Practical Gas Chromatography, Berlin, Heidelberg: Springer-Verlag; 2014. p 529-575.

32. Grob K. Split and splitless injection for quantitative gas chromatography. Weinheim-Germany: Wiley-VCH; 2001. $460 \mathrm{p}$. 33. Mayfield KJ, Shalliker RA, Catchpoole HJ, Sweeney AP, Wong $\mathrm{V}$, Guiochon $\mathrm{G}$. Viscous fingering induced flow instability in multidimensional liquid chromatography. $J$ Chromatogr $A$. 2005;1080:124-131.
34. Rousseaux G, De Wit A, Martin M. Viscous fingering in packed chromatographic columns: Linear stability analysis. $J$ Chromatogr A. 2007;1149:254-273.

35. Enmark M, Åsberg D, Shalliker A, Samuelsson J, Fornstedt T. A closer study of peak distortions in supercritical fluid chromatography as generated by the injection. J Chromatogr $A$. 2015;1400:131-139.

36. Samuelsson J, Fornstedt T, Shalliker A. Exogenous factors contributing to column bed heterogeneity. Part 1: Consequences of "air" injections in liquid chromatography. J Chromatogr A. 2015;1406:186-191.

37. Shalliker RA, Samuelsson J, Fornstedt T. Sample introduction for high performance separations. TrAC Trends Anal Chem. 2016;81:34-41.

38. Shalliker RA, Guiochon G. Understanding the importance of the viscosity contrast between the sample solvent plug and the mobile phase and its potential consequence in two-dimensional high-performance liquid chromatography. J Chromatogr A.

2009;1216:787-793.

39. Aragón A. Toledano RM, Gea S, Cortés JM, Vázquez A Villén J. Large volume injection in gas chromatography using the through oven transfer adsorption desorption interface operating under vacuum. Talanta. 2014;123:39-44

40. Aragón A. Toledano RM, Cortés JM, Vázquez A, Villén J. Use of nitrogen to remove solvent from through oven transfer adsorption desorption interface during analysis of polycyclic aromatic hydrocarbons by large volume injection in gas chromatography. J Chromatogr A. 2014;1339:224-228.

41. Aragón A. Toledano RM, Vázquez A, Villén J, Cortés JM. Analysis of polycyclic aromatic hydrocarbons in aqueous samples by large volume injection gas chromatography-mass spectrometry using the through oven transfer adsorption desorption interface. Talanta. 2015;139:1-5. 